\section{(1)}

CrossMark

\title{
Risk assessment in medically treated chronic thromboembolic pulmonary hypertension patients
}

\author{
Marion Delcroix (1) ${ }^{1,2}$, Gerd Staehler ${ }^{3}$, Henning Gall $\mathbb{B}^{4}$, Ekkehard Grünig ${ }^{5}$, \\ Matthias Held ${ }^{6}$, Michael Halank ${ }^{7}$, Hans Klose ${ }^{8}$, Anton Vonk-Noordegraaf ${ }^{9}$, \\ Stephan Rosenkranz ${ }^{10}$, Joanna Pepke-Zaba ${ }^{11}$, Christian F. Opitz ${ }^{12,13}$, \\ J. Simon R. Gibbs ${ }^{14}$, Tobias J. Lange ${ }^{15}$, Iraklis Tsangaris ${ }^{16}$, Doerte Huscher ${ }^{17}$, \\ David Pittrow (1) ${ }^{18}$, Karen M. Olsson ${ }^{19}$ and Marius M. Hoeper (1) ${ }^{19}$
}

@ERSpublications

The risk stratification for PAH of the current European PH guidelines may allow survival prediction in medically treated CTEPH patients http://ow.ly/zx54301I41T

Cite this article as: Delcroix M, Staehler G, Gall H, et al. Risk assessment in medically treated chronic thromboembolic pulmonary hypertension patients. Eur Respir J 2018; 52: 1800248 [https://doi.org/10.1183/ 13993003.00248-2018].

ABSTRACT Abbreviated versions of the risk stratification strategy of the European Society of Cardiology (ESC)/European Respiratory Society (ERS) pulmonary hypertension guidelines have been recently validated in patients with pulmonary arterial hypertension. We aimed to investigate their prognostic value in medically treated chronic thromboembolic pulmonary hypertension (CTEPH) patients from the COMPERA registry, which collects six variables of interest (World Health Organization Functional Class, 6-min walk distance, brain natriuretic peptide, right atrial pressure, cardiac index and mixed venous oxygen saturation).

We included patients with at least one follow-up visit, no pulmonary endarterectomy and at least three of the six variables available, and classified the patients into low-, intermediate- and high-risk groups. As a secondary analysis, the number of noninvasive low-risk criteria was counted. The association between risk assessment and survival was evaluated.

Data from inclusion and follow-up (median 7 months) visits were available for 561 and 231 patients, respectively. Baseline 1 - and 5-year survival estimates were significantly different $(\mathrm{p}<0.0001)$ in the baseline low-risk (98.6\% and $88.3 \%$, respectively), intermediate-risk (94.9\% and $61.8 \%$, respectively) and high-risk (75.5\% and $32.9 \%$, respectively) cohorts. Follow-up data were even more discriminative, with $100 \%, 92 \%$ and $69 \%$ 1-year survival, respectively. The number of low-risk noninvasive criteria was also associated with survival.

These analyses suggest that the ESC/ERS risk assessment may be applicable in patients with medically treated CTEPH. 
Affiliations: 'Dept of Respiratory Diseases, University Hospitals of Leuven, Leuven, Belgium. ${ }^{2}$ Respiratory Division, Dept CHROMETA, KU Leuven - University of Leuven, Leuven, Belgium. ${ }^{3}$ Medical Clinic I, Clinic Loewenstein, Loewenstein, Germany. "Dept of Pneumology, University of Giessen and Marburg, Giessen, Germany. ${ }^{5}$ Thoraxclinic at the University Hospital Heidelberg and German Center of Lung Research (DZL), Heidelberg, Germany. ${ }^{6}$ Dept of Internal Medicine, Respiratory Medicine and Cardiology, Mission Medical Hospital, Würzburg, Germany. ${ }^{7}$ Internal Medicine I, University Hospital Carl Gustav Carus of the Technical University Dresden, Dresden, Germany. ${ }^{8}$ Dept of Respiratory Medicine, Center of Oncology, University Medical Center Hamburg-Eppendorf, Hamburg, Germany. ${ }^{9}$ Dept of Pulmonary Diseases, VU University Medical Center, Amsterdam, The Netherlands. ${ }^{10} \mathrm{Clinic}$ III for Internal Medicine (Cardiology) and Center for Molecular Medicine (CMMC), and the Cologne Cardiovascular Research Center (CCRC), University of Cologne, Cologne, Germany. ${ }^{11}$ Pulmonary Vascular Diseases Unit, Royal Papworth Hospital, Cambridge, UK. ${ }^{12}$ Dept of Cardiology, DRK Kliniken Berlin, Berlin, Germany. ${ }^{13}$ Dept of Cardiology, Medical University of Greifswald, Greifswald, Germany. ${ }^{14}$ Dept of Cardiology, National Heart and Lung Institute, Imperial College London, London, UK. ${ }^{15}$ Dept of Internal Medicine II, Division of Pneumology, University Medical Center Regensburg, Regensburg, Germany. ${ }^{16}$ Pulmonary Hypertension Clinic, Attikon University Hospital, National and Kapodistrian University of Athens, Athens, Greece. ${ }^{17}$ Epidemiology Unit, German Rheumatism Research Centre, Berlin, Germany. ${ }^{18}$ Institute for Clinical Pharmacology, Medical Faculty, Technical University, Dresden, Germany. ${ }^{19}$ Dept of Respiratory Medicine, Hannover Medical School and German Center of Lung Research (DZL), Hannover, Germany.

Correspondence: Marion Delcroix, Dept of Respiratory Diseases, University Hospitals of Leuven, Herestraat 49, 3000 Leuven, Belgium. E-mail marion.delcroix@uzleuven.be

\section{Introduction}

The 2015 European Society of Cardiology (ESC)/European Respiratory Society (ERS) pulmonary hypertension guidelines recommend to evaluate the severity of patients with pulmonary arterial hypertension (PAH) with a panel of data derived from clinical assessment, exercise tests, biochemical markers, and echocardiographic and haemodynamic evaluations, with regular follow-up assessments every 3-6 months in stable patients [1]. The resulting data should be used to categorise patients into risk groups with low risk (estimated 1-year mortality rate $<5 \%)$, intermediate risk $(5-10 \%)$ and high risk $(>10 \%)$, and to facilitate treatment decision in a treat-to-target approach. The accuracy of this risk assessment strategy, at baseline as well as during follow-up, has been recently demonstrated under real-life conditions in three different prospective cohorts: the Swedish Pulmonary Hypertension Registry (SPAHR) [2], the French Pulmonary Hypertension Registry [3] and COMPERA (Comparative, Prospective Registry of Newly Initiated Therapies for Pulmonary Hypertension) [4].

There is as yet no established risk assessment strategy to guide treatment decisions in patients with inoperable chronic thromboembolic pulmonary hypertension (CTEPH). Here, we aimed to investigate whether the ESC/ERS risk stratification strategy could also be applied in patients with CTEPH who were not candidates for surgery, by analysing data from COMPERA, a European-based pulmonary hypertension registry that captures data from patients with all forms of pulmonary hypertension who receive targeted medical therapy $[5,6]$. We applied two sets of analyses, looking at the discriminative value of global low-, intermediate- and high-risk scores, as had been done in the SPAHR and the COMPERA analysis [2, 4], and at the number of low-risk criteria, following the French strategy [3], to predict long-term prognosis.

\section{Methods \\ Database}

COMPERA (www.COMPERA.org; registered at Clinicaltrials.gov with identifier NCT01347216) is an ongoing web-based pulmonary hypertension registry launched in 2007 that collects baseline, follow-up and outcome data from patients who receive PAH medical therapies. Initially, COMPERA included only patients with $\mathrm{PAH}$; however, since 2009 the registry has captured patients with all forms of pulmonary hypertension, including CTEPH. Specialised centres in several European countries participate (Austria, Belgium, Germany, Greece, Hungary, Italy, Netherlands, Switzerland and the UK), with 80\% of the patients coming from German pulmonary hypertension centres. COMPERA enrols only patients with newly diagnosed pulmonary hypertension, i.e. patients must be entered into the database $\leqslant 6$ months after the date of diagnosis. Further methodological details have been published elsewhere [4-6].

Among other variables, World Health Organization Functional Class (WHO FC), 6-min walk distance (6MWD), brain natriuretic peptide (BNP or N-terminal pro-BNP (NT-proBNP)), right atrial pressure (RAP), cardiac index (CI) and mixed venous oxygen saturation $\left(\mathrm{S}_{\mathrm{vO}}\right)$ are captured in COMPERA whenever available. These six variables were used in the present study for the validation of a truncated version of the risk assessment strategy proposed by the ESC/ERS pulmonary hypertension guidelines [1]. 
Patients

Patients were selected from the COMPERA database according to the following criteria: 1) treatment-naive patients newly diagnosed with CTEPH or residual pulmonary hypertension after pulmonary endarterectomy (PEA) between January 1, 2009 and December 2, 2017, with data from baseline and at least one follow-up visit available; 2) mean pulmonary artery pressure $\geqslant 25 \mathrm{mmHg}$ and pulmonary artery wedge pressure $\leqslant 15 \mathrm{mmHg}$ at the time of diagnosis; 3) no PEA or balloon pulmonary angioplasty (BPA) during follow-up; and 4) at least three of the six listed variables available at baseline.

\section{Risk stratification strategy}

An abbreviated version of the 2015 ESC/ERS risk stratification strategy, including the six variables recorded in COMPERA, was used to categorise patients as low, intermediate or high risk. Following a validation strategy proposed by KylHammar et al. [2], the cut-off values proposed in the guidelines were graded 1 (low risk), 2 (intermediate risk) or 3 (high risk). For each patient, the sum of all grades was divided by the number of available variables and rounded to the next integer to define the risk group. Calculations were made from baseline assessments and from follow-up assessments between 3 months and 2 years after the initiation of PAH medical therapy.

In a second set of analyses, proposed by Boucly et al. [3], we evaluated the presence of three noninvasive low-risk criteria ("risk score-3") that were defined as 1) WHO FC I or II, 2) 6MWD >440 m and 3) BNP $<50 \mathrm{ng} \cdot \mathrm{L}^{-1}$ or NT-proBNP $<300 \mathrm{ng} \cdot \mathrm{L}^{-1}$. Patients were classified according to the number of low-risk criteria present at inclusion and at the time of follow-up.

\section{Statistical analyses}

The primary analysis set consisted of the entire patient population that fulfilled the inclusion criteria listed earlier. Sensitivity analyses were performed with those patients for whom all six risk score variables were available and for the subgroup of patients with surgically inoperable CTEPH. Other subgroups were not assessed because the numbers of patients were considered too low.

For the follow-up risk stratification, patients who underwent their first comprehensive follow-up risk assessment between 3 months and 2 years after treatment initiation were considered. Where available, we chose the first visit that included follow-up haemodynamics. If no haemodynamic follow-up was available during the first 2 years after diagnosis, we selected the follow-up visit that contained most of

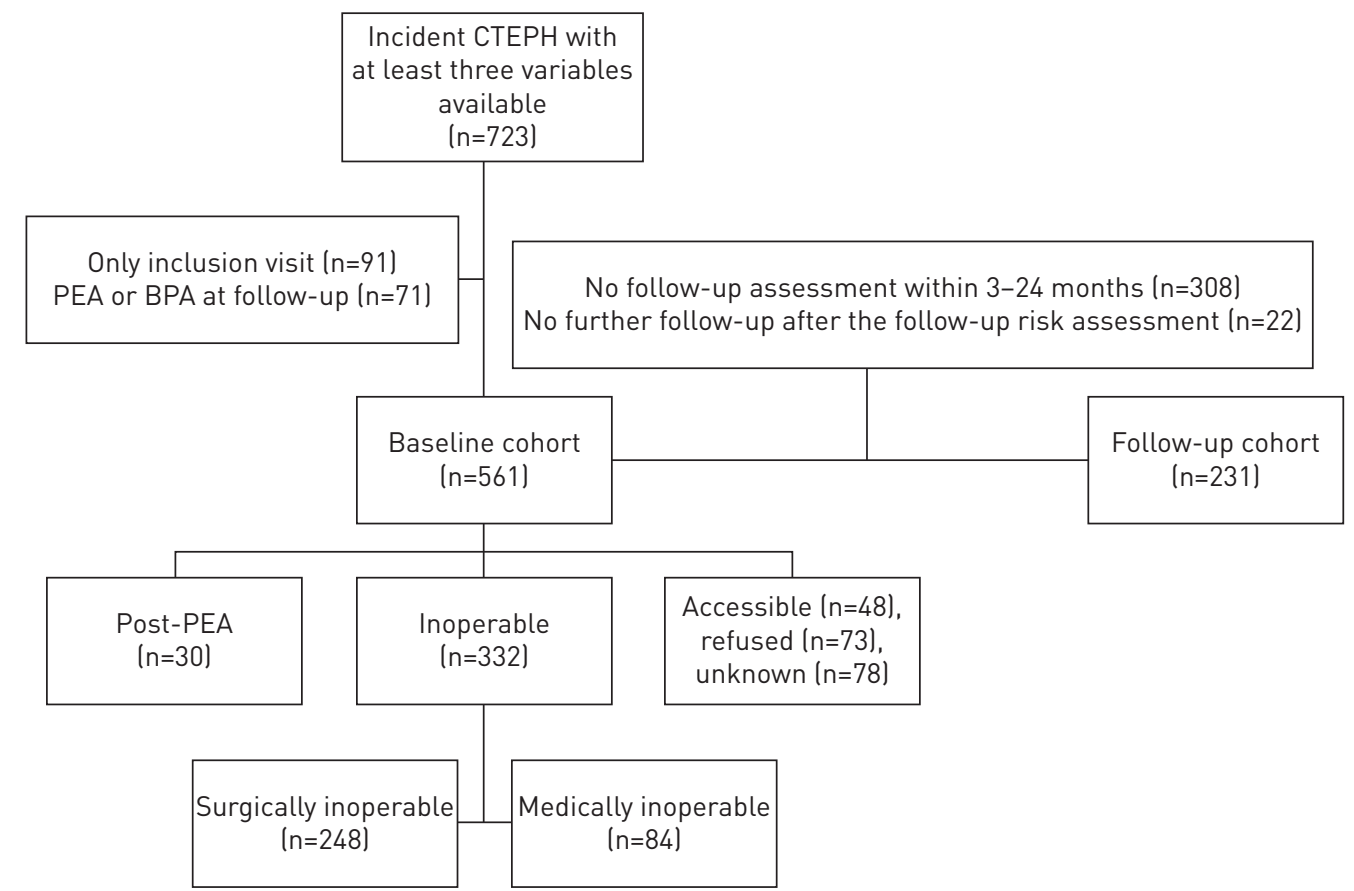

FIGURE 1 Patient disposition. CTEPH: chronic thromboembolic pulmonary hypertension; PEA: pulmonary endarterectomy; BPA: balloon pulmonary angioplasty. 
the data of interest. For all analyses, only patients with at least one further follow-up were included in the analysis.

Continuous data are presented as mean with standard deviation or as median and interquartile range (IQR). In patients who died, investigators were asked to provide the most likely cause of death. There was no independent adjudication of causes of death. Survival was evaluated using Kaplan-Meier analysis and the log-rank test, truncated at 5 years. Survival was censored at the last available visit reported for a patient; mortality was recorded with the date of the patient's death. Hazard ratios for the single risk score items were estimated using univariate and multivariate Cox regression analysis, using the respective low-risk group as reference. SPSS Statistics version 19.0 (IBM, Armonk, NY, USA) was used for analysis.

\begin{tabular}{|c|c|c|c|c|c|}
\hline Patients & & $561(100)$ & $81(14)$ & $382(68)$ & $98(18)$ \\
\hline Age years & & $69 \pm 13$ & $63 \pm 12$ & $70 \pm 12$ & $71 \pm 12$ \\
\hline Female & & 54 & 49 & 55 & 52 \\
\hline Post-PEA & & $30(5)$ & $9(11)$ & $20(5)$ & $1(1)$ \\
\hline Surgically inoperable & & $248(44)$ & $37(46)$ & $176(46)$ & $35(36)$ \\
\hline Medically inoperable & & $84(15)$ & $13(16)$ & $53(14)$ & $18(18)$ \\
\hline Accessible & & $48(9)$ & $6(7)$ & $31(8)$ & $11(11)$ \\
\hline Refused surgery & & $73(13)$ & $6(7)$ & $51(13)$ & $16(16)$ \\
\hline Operability unknown & & $78(14)$ & $10(12)$ & $51(13)$ & $17(17)$ \\
\hline $\mathrm{RAP} \mathrm{mmHg}$ & 533 & $8 \pm 5$ & $5 \pm 3$ & $8 \pm 5$ & $13 \pm 5$ \\
\hline mPAP mmHg & & $42 \pm 11$ & $36 \pm 9$ & $42 \pm 11$ & $48 \pm 11$ \\
\hline PAWP mmHg & & $9 \pm 4$ & $9 \pm 3$ & $9 \pm 4$ & $10 \pm 3$ \\
\hline $\mathrm{Cl} \mathrm{L} \cdot \mathrm{min}^{-1} \cdot \mathrm{m}^{-2}$ & 525 & $2.2 \pm 0.8$ & $3.0 \pm 0.7$ & $2.2 \pm 0.7$ & $1.6 \pm 0.3$ \\
\hline PVR dyn $\cdot \mathrm{s} \cdot \mathrm{cm}^{-5}$ & 547 & $734 \pm 387$ & $411 \pm 177$ & $702 \pm 315$ & $1135 \pm 442$ \\
\hline $\mathrm{SvO}_{2} \%$ & 508 & $63 \pm 9$ & $71 \pm 5$ & $63 \pm 8$ & $53 \pm 7$ \\
\hline \multicolumn{6}{|l|}{ Comorbidities } \\
\hline Any comorbidities & 478 & 91 & 90 & 91 & 93 \\
\hline $\mathrm{CHD}$ & 449 & 20 & 11 & 21 & 23 \\
\hline AHT & 460 & 59 & 55 & 61 & 56 \\
\hline Diabetes mellitus & 462 & 16 & 9 & 16 & 23 \\
\hline OSAS & 418 & 9 & 14 & 9 & 4 \\
\hline PCA & & 1 & 0 & 1 & 3 \\
\hline Monotherapy & & 93 & 93 & 95 & 90 \\
\hline Combination therapy & & 7 & 7 & 5 & 10 \\
\hline Anticoagulation & & $97^{\uparrow}$ & 98 & 96 & 97 \\
\hline
\end{tabular}

Data are presented as $\mathrm{n}(\%)$, mean \pm SD, $\%$ or median (interquartile range), unless otherwise stated. BMI: body mass index; CTEPH: chronic thromboembolic pulmonary hypertension; PEA: pulmonary endarterectomy; WHO FC: World Health Organization Functional Class; 6MWD: 6-min walk distance; BNP: brain natriuretic peptide; NT-proBNP: N-terminal pro-BNP; RAP: right atrial pressure; mPAP: mean pulmonary arterial pressure; PAWP: pulmonary arterial wedge pressure; $\mathrm{Cl}$ : cardiac index; PVR: pulmonary vascular resistance; Svo ${ }_{2}$ : mixed venous oxygen saturation; CHD: coronary heart disease; AHT: arterial hypertension; OSAS: obstructive sleep apnoea; VTE: venous thromboembolism; ERA: endothelin receptor antagonist ( $87 \%$ bosentan); PDE5i: phosphodiesterase-5 inhibitor (72\% sildenafil); sGCS: soluble guanylate cyclase stimulator; PCA: prostacyclin analogue. \#: $n$ is specified when data are not available for the whole population; ${ }^{\text {: }}: 26 \%$ direct oral anticoagulants. 
a)

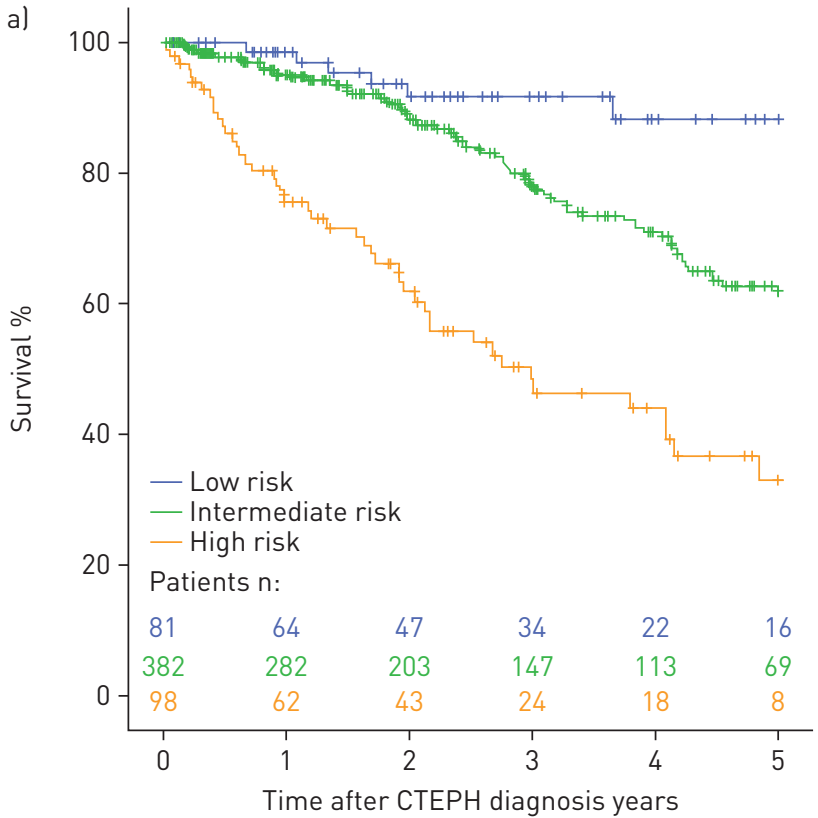

\begin{tabular}{|l|cccccc|}
\hline \multirow{2}{*}{ Survival \% } & \multicolumn{6}{|c|}{ Years after enrolment } \\
\cline { 2 - 7 } & 0 & 1 & 2 & 3 & 4 & 5 \\
\hline Low risk & 100 & 98.6 & 91.7 & 91.7 & 88.3 & 88.3 \\
Intermediate risk & 100 & 94.9 & 88.2 & 78.4 & 71.0 & 61.8 \\
High risk & 100 & 75.5 & 61.9 & 48.3 & 44.1 & 32.9 \\
\hline
\end{tabular}

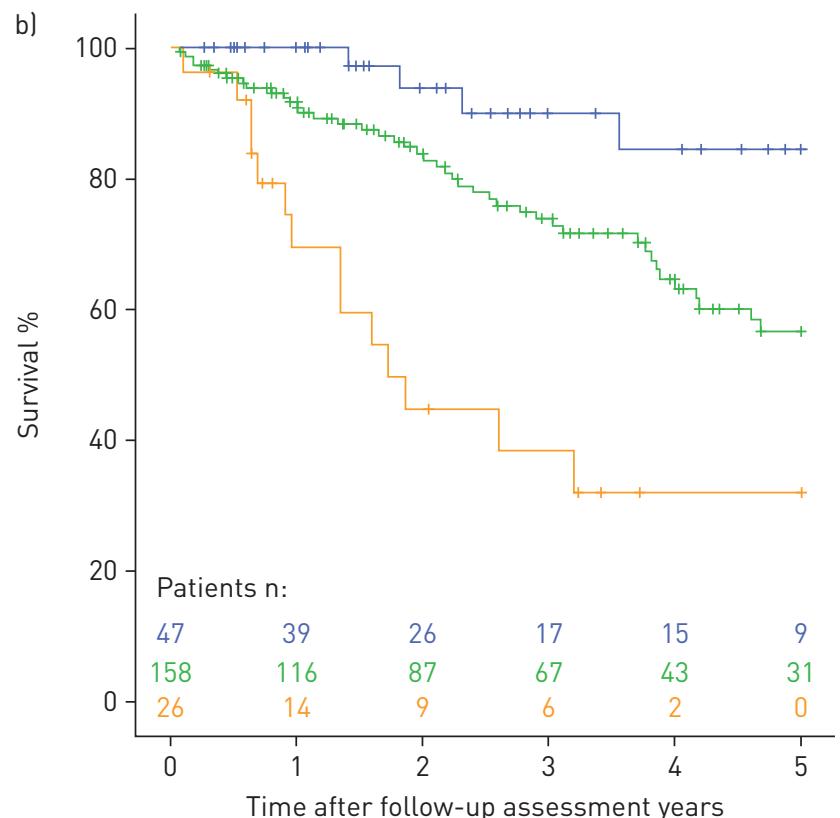

\begin{tabular}{|l|cccccc|}
\hline \multirow{2}{*}{ Survival \% } & \multicolumn{7}{|c|}{ Years after enrolment } \\
\cline { 2 - 7 } & 0 & 1 & 2 & 3 & 4 & 5 \\
\hline Low risk & 100 & 100 & 93.9 & 90.0 & 84.3 & 84.3 \\
Intermediate risk & 100 & 91.6 & 83.8 & 73.8 & 63.1 & 56.5 \\
High risk & 100 & 69.4 & 44.6 & 38.3 & 31.9 & \\
\hline
\end{tabular}

FIGURE 2 Five-year survival (at least three variables available): a) from baseline and b) from follow-up. CTEPH: chronic thromboembolic pulmonary hypertension.

\section{Results}

Risk stratification at baseline and mortality

A total of 561 patients met the inclusion criteria of newly diagnosed CTEPH or residual pulmonary hypertension after PEA, with at least one follow-up visit, no PEA or BPA during follow-up and at least three of the six risk score variables available at baseline (figure 1). At the time of inclusion, $44.2 \%$ of these patients were inoperable due to peripheral location of the thrombus (surgically inoperable) and $15.0 \%$ due to comorbidities (medically inoperable), $8.6 \%$ had surgically accessible disease, $13.0 \%$ had refused PEA, and $5.3 \%$ had persistent pulmonary hypertension after pulmonary endarterectomy (post-PEA); operability was still under investigation or information was not available (unknown) for $13.9 \%$. All patients received PAH medical therapies within 6 months of study inclusion, since the start of PAH therapy is an inclusion criterion for COMPERA. Patient characteristics are shown in table 1. It is noteworthy that the low-risk group was younger and included more post-PEA patients. The increasing mortality risk was accompanied by an increase in comorbidities such as diabetes mellitus and atrial fibrillation, while obstructive sleep apnoea and thyroid disease tended to decrease.

Out of the six variables of interest for this study, at least two were available in 568 patients, at least three in 561 (98.8\%) patients (baseline analysis set), at least four in 537 (94.5\%) patients, at least five in 482 $(84.9 \%)$ patients and all six variables were available in $318(56.0 \%)$ patients. WHO FC was available in $97.7 \%$ of the patients, $6 \mathrm{MWD}$ in $79.4 \%$, BNP or NT-proBNP in $80.3 \%$, RAP in $94.4 \%$, CI in $92.8 \%$ and $\mathrm{SvO}_{2}$ in $89.6 \%$.

During the follow-up, up to 5 years after diagnosis, 132 patients $(23.5 \%)$ died: six $(7.4 \%)$ in the low-risk cohort, $80(20.9 \%)$ in the intermediate-risk cohort and $46(46.9 \%)$ in the high-risk cohort. Right heart failure was reported as the most likely cause of death in $54 \%$ of all patients: $50 \%$ of the low-risk group, $46 \%$ of the intermediate-risk group and $67 \%$ of the high-risk group. Infection (27.4\%), cancer (18.3\%) and bleeding (13.7\%) were other frequent causes of death. In addition, 14 (2.5\%) patients were lost to follow-up: one (1.2\%) in the low-risk group, nine (2.4\%) in the intermediate-risk group and four $(4.1 \%)$ in the high-risk group.

For the entire baseline cohort, the survival estimates at 1, 2, 3, 4 and 5 years were $92.0 \%, 83.9 \%, 74.7 \%$, $68.3 \%$ and $59.8 \%$, respectively. The corresponding survival estimates for the low-, intermediate- and 
high-risk groups are presented in figure $2 \mathrm{a}(\mathrm{p}<0.0001$ for all-groups comparison, with $\mathrm{p}=0.007$ for lowversus intermediate-risk group comparison and $\mathrm{p}<0.0001$ for intermediate- versus high-risk group comparison). The predictive value of each variable at baseline is shown in figure 3 .

Similar results were obtained from a sensitivity analysis that included only those 318 patients for whom all six baseline variables were available. Here, the survival differences between the three risk categories were also statistically significant $(\mathrm{p}<0.0001$ for all-groups comparison, with $\mathrm{p}=0.032$ for low- versus intermediate-risk group comparison and $\mathrm{p}<0.0001$ for intermediate- versus high-risk group comparison) (supplementary table S1 and supplementary figure S1). The results of the analysis for the surgically inoperable CTEPH subgroup $(n=248)$ are shown in supplementary table S2 and figure 4. The survival differences between all three groups were statistically significant $(\mathrm{p}<0.0001$ for all-groups comparison, with $\mathrm{p}=0.011$ for low- versus intermediate-risk group comparison and $\mathrm{p}<0.0001$ for intermediate- versus high-risk group comparison).

\section{Risk stratification at follow-up and mortality}

Out of the 496 patients with follow-up data within 2 years of treatment initiation, at least two variables were available in $435(87.7 \%)$ patients, at least three in $253(51.0 \%)$ patients, at least four in $100(20.2 \%)$ patients, at least five in $78(15.7 \%)$ patients and all six in $44(8.9 \%)$ patients. WHO FC was available in $86.3 \%$ of the patients, $6 \mathrm{MWD}$ in $72.4 \%$, BNP or NT-proBNP in $68.1 \%$, RAP in $20.8 \%$, CI in $20.8 \%$ and $\mathrm{SvO}_{2}$ in $19.8 \%$.

Follow-up data (between 3 months and 2 years after treatment initiation) with at least three out of the six variables of interest and at least one follow-up thereafter were available for 231 patients (figure 1), with a median (IQR) duration between inclusion and follow-up risk assessment of 7 (4-10) months. The characteristics of these patients at the time of follow-up risk assessment are shown in table 2 and supplementary table S3.

One $(0.4 \%)$ patient was lost to follow-up in the intermediate-risk group. Within 5 years of follow-up assessment, 61 patients (26.4\%) had died: four (8.5\%) in the low-risk group, $43(27.2 \%)$ in the intermediate-risk group and $14(53.8 \%)$ in the high-risk group. The survival estimates at 1, 2, 3, 4 and

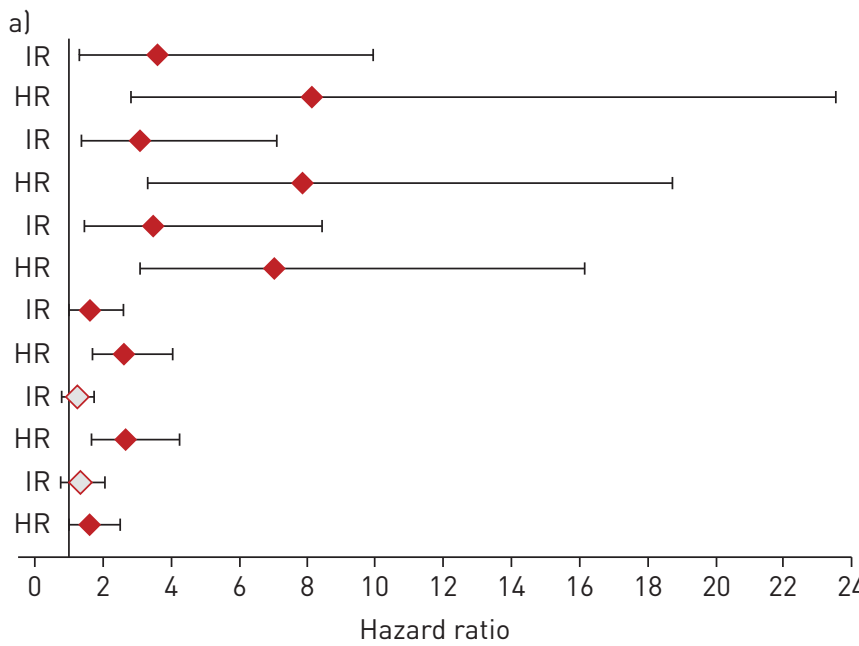

\begin{tabular}{|c|c|c|}
\hline \multirow{2}{*}{ 6MWD } & IR & $3.63(1.33-9.93)$ \\
\hline & $\mathrm{HR}$ & $8.17(2.84-23.50)$ \\
\hline \multirow{2}{*}{ WHO FC } & IR & $3.11(1.36-7.12)$ \\
\hline & $\mathrm{HR}$ & $7.87(3.31-18.70)$ \\
\hline \multirow{2}{*}{$\begin{array}{l}\text { BNP/ } \\
\text { NT-proBNP }\end{array}$} & IR & $3.51(1.46-8.40)$ \\
\hline & $\mathrm{HR}$ & $7.05(3.07-16.17)$ \\
\hline \multirow{2}{*}{$\mathrm{SvO}_{2}$} & IR & $1.62(1.01-2.59)$ \\
\hline & $\mathrm{HR}$ & $2.61(1.69-4.04)$ \\
\hline \multirow{2}{*}{ RAP } & IR & $1.17(0.79-1.72)$ \\
\hline & $\mathrm{HR}$ & $2.65(1.65-4.25)$ \\
\hline \multirow{2}{*}{$\mathrm{Cl}$} & IR & $1.25(0.75-2.07)$ \\
\hline & $\mathrm{HR}$ & $1.62(1.04-2.52)$ \\
\hline
\end{tabular}

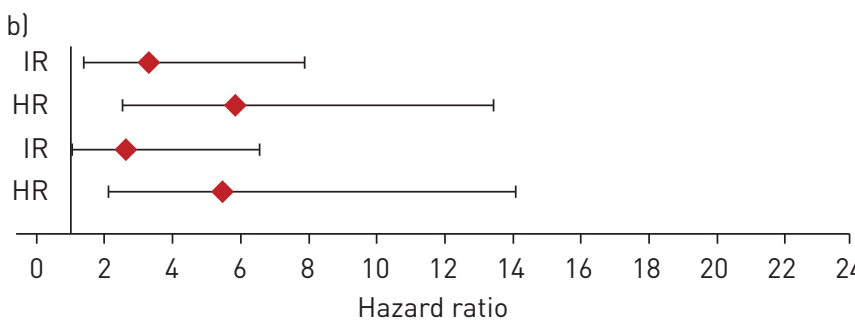

\begin{tabular}{|l|l|l|}
\hline \multirow{2}{*}{$\begin{array}{l}\text { BNP/ } \\
\text { NT-proBNP }\end{array}$} & IR & $3.29(1.37-7.90)$ \\
\cline { 2 - 3 } & $H R$ & $5.82(2.53-13.41)$ \\
\hline \multirow{2}{*}{ WHO FC } & IR & $2.64(1.07-6.54)$ \\
\cline { 2 - 3 } & $H R$ & $5.46(2.12-14.07)$ \\
\hline
\end{tabular}

FIGURE 3 Hazard ratios (95\% confidence intervals) for the risk score items at baseline, estimated by a) univariate and b) multivariate Cox regression analysis, using the respective low-risk group as reference. IR: intermediate-risk group; HR: high-risk group; 6MWD: 6-min walk distance; WHO FC: World Health Organization Functional Class; BNP: brain natriuretic peptide; NT-proBNP: N-terminal pro-BNP; $\mathrm{SvO}_{2}$ : mixed venous oxygen saturation; RAP: right atrial pressure; $\mathrm{Cl}$ : cardiac index. Grey symbols indicate values not different from 1. 
FIGURE 4 Five-year survival from baseline of surgically inoperable CTEPH (at least three variables).

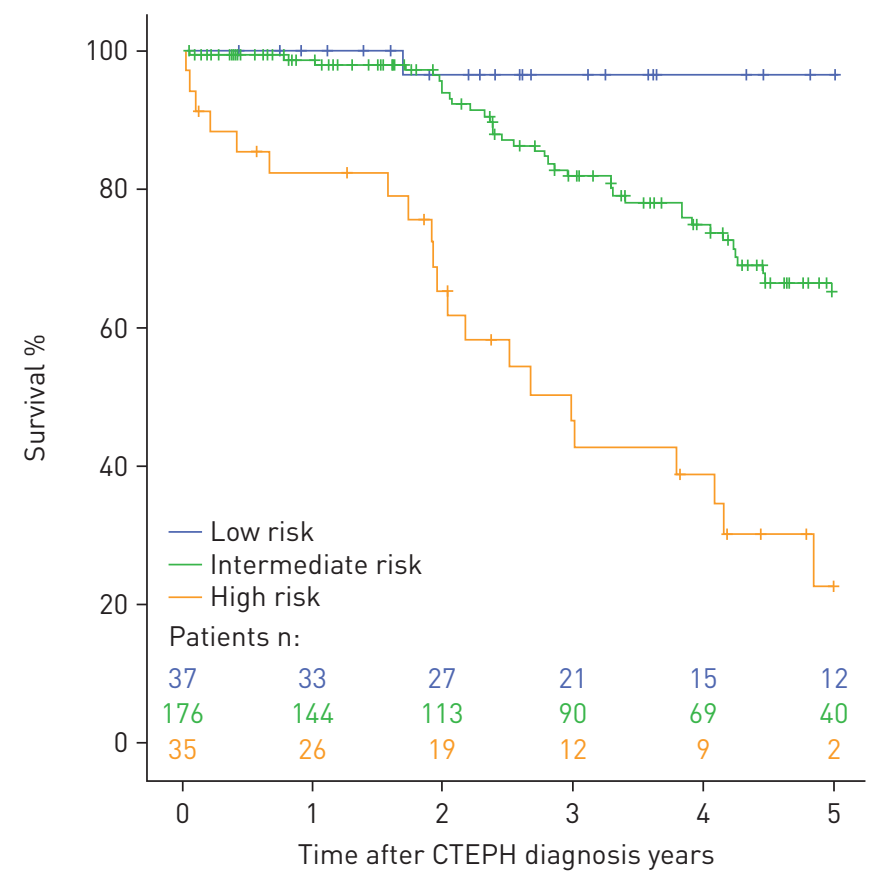

\begin{tabular}{|l|cccccc|}
\hline \multirow{2}{*}{ Survival \% } & \multicolumn{6}{|c|}{ Years after enrolment } \\
\cline { 2 - 7 } & 0 & 1 & 2 & 3 & 4 & 5 \\
\hline Low risk & 100 & 100 & 96.6 & 96.6 & 96.6 & 96.6 \\
Intermediate risk & 100 & 98.8 & 93.9 & 81.9 & 74.9 & 64.9 \\
High risk & 100 & 82.4 & 65.4 & 46.5 & 38.8 & 22.6 \\
\hline
\end{tabular}

5 years for the low-, intermediate- and high-risk groups are presented in figure $2 b \quad(p<0.0001$ for all-groups comparison, with $\mathrm{p}=0.014$ for low- versus intermediate-risk group comparison and $\mathrm{p}=0.0001$ for intermediate- versus high-risk group comparison).

The full risk score analysis at follow-up could not be performed because of the low case numbers, since right heart catheterisation was not done regularly at follow-up.

From baseline to follow-up, 50 out of 231 (21.6\%) patients improved their risk category; 152 out of 231 $(65.8 \%)$ patients remained stable and 29 out of $231(12.6 \%)$ patients deteriorated. Changes in the risk category from baseline to follow-up were associated with a shift in the mortality risk $(p<0.0001)$ as shown in figure 5. The groups that worsened from "low" to "high" risk $(\mathrm{n}=1)$ and that improved from "high" to "low" risk $(\mathrm{n}=3)$ were omitted from the survival analysis.

\section{"Risk score-3" approach at baseline and at follow-up}

At baseline, the three noninvasive variables, i.e. WHO FC, 6MWD and BNP/NT-proBNP, were available in 368 patients, and $64.1 \%, 23.1 \%, 9.5 \%$ and $3.3 \%$ of them had no, one, two or three low-risk criteria, respectively. Patient characteristics are presented in supplementary table S4. It is noteworthy that age progressively decreased with increasing number of low-risk criteria; female prevalence also decreased. Comorbidities were randomly distributed among the risk groups. The survival estimates at 1,2, 3, 4 and 5 years for no, one, two and three low-risk criteria are presented in figure $6 \mathrm{a}(\mathrm{p}<0.0001$ for all-groups comparison, with significant differences between none and one, two or three low-risk criteria).

At follow-up, the three variables were available in 199 patients, and $47.7 \%, 32.2 \%, 11.6 \%$ and $8.5 \%$ of them had no, one, two or three low-risk criteria, respectively. Patient characteristics are presented in supplementary table S5. The survival estimates at 1,2, 3, 4 and 5 years for none, one, two and three low-risk criteria are presented in figure $6 \mathrm{~b}$ ( $\mathrm{p}=0.017$ for all-groups comparison, with significant differences between none and two or three low-risk criteria).

\section{Discussion}

To the best of our knowledge, the present analyses concern one of the largest prospectively collected contemporary populations of newly diagnosed CTEPH patients, not operated on during follow-up. The 
TABLE 2 Variables obtained between 3 months and 2 years after treatment initiation of the patients included in the follow-up risk stratification groups

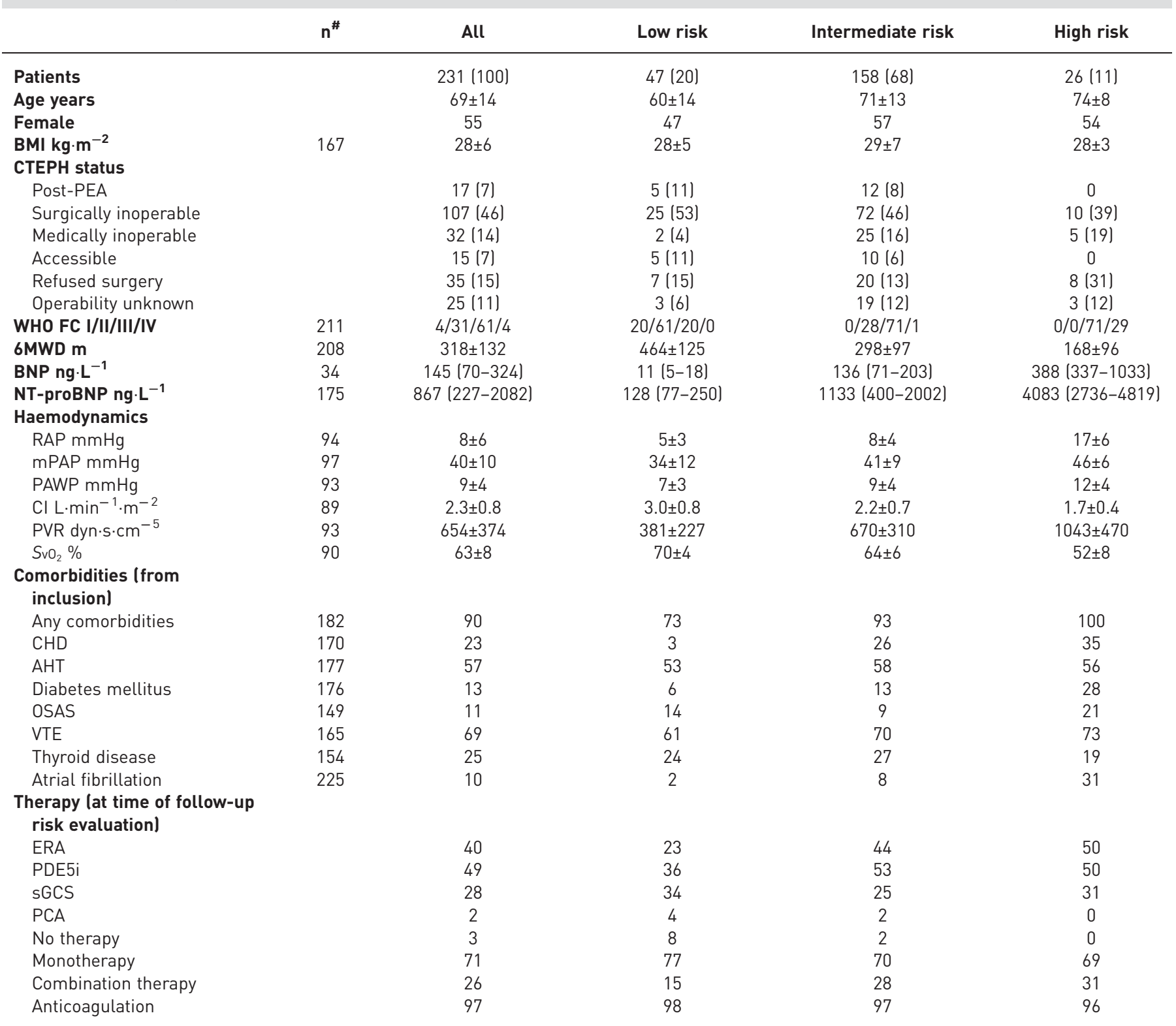

Data are presented as $\mathrm{n}(\%)$, mean $\pm \mathrm{SD}, \%$ or median (interquartile range), unless otherwise stated. BMI: body mass index; CTEPH: chronic thromboembolic pulmonary hypertension; PEA: pulmonary endarterectomy; WHO FC: World Health Organization Functional Class; 6MWD: 6-min walk distance; BNP: brain natriuretic peptide; NT-proBNP: N-terminal pro-BNP; RAP: right atrial pressure; mPAP: mean pulmonary arterial pressure; PAWP: pulmonary arterial wedge pressure; $\mathrm{Cl}$ : cardiac index; PVR: pulmonary vascular resistance; Svo ${ }_{2}$ mixed venous oxygen saturation; CHD: coronary heart disease; AHT: arterial hypertension; OSAS: obstructive sleep apnoea; VTE: venous thromboembolism; ERA: endothelin receptor antagonist; PDE5i: phosphodiesterase-5 inhibitor; sGCS: soluble guanylate cyclase stimulator; PCA: prostacyclin analogue. ${ }^{\#}: \mathrm{n}$ is specified when data are not available for the whole population.

main findings can be summarised as follows: 1) overall survival estimates of $92 \%, 75 \%$ and $60 \%$ at 1,3 and 5 years, respectively; 2) an efficient risk stratification of 5 -year mortality at baseline and at follow-up using an abbreviated ESC/ERS risk score assessment; and 3) confirmation of the risk estimates proposed in the ESC/ERS pulmonary hypertension guidelines with 1 -year mortality risks of $<5 \%, 5-10 \%$ and $>10 \%$ in patients at low, intermediate or high risk, respectively, for the global cohort of medically treated CTEPH patients, both at baseline as well as at follow-up.

The survival estimates observed in this series were comparable with findings of the European CTEPH registry [7], in which a 3-year survival of $70 \%$ was observed in a cohort of 275 nonoperated patients with 

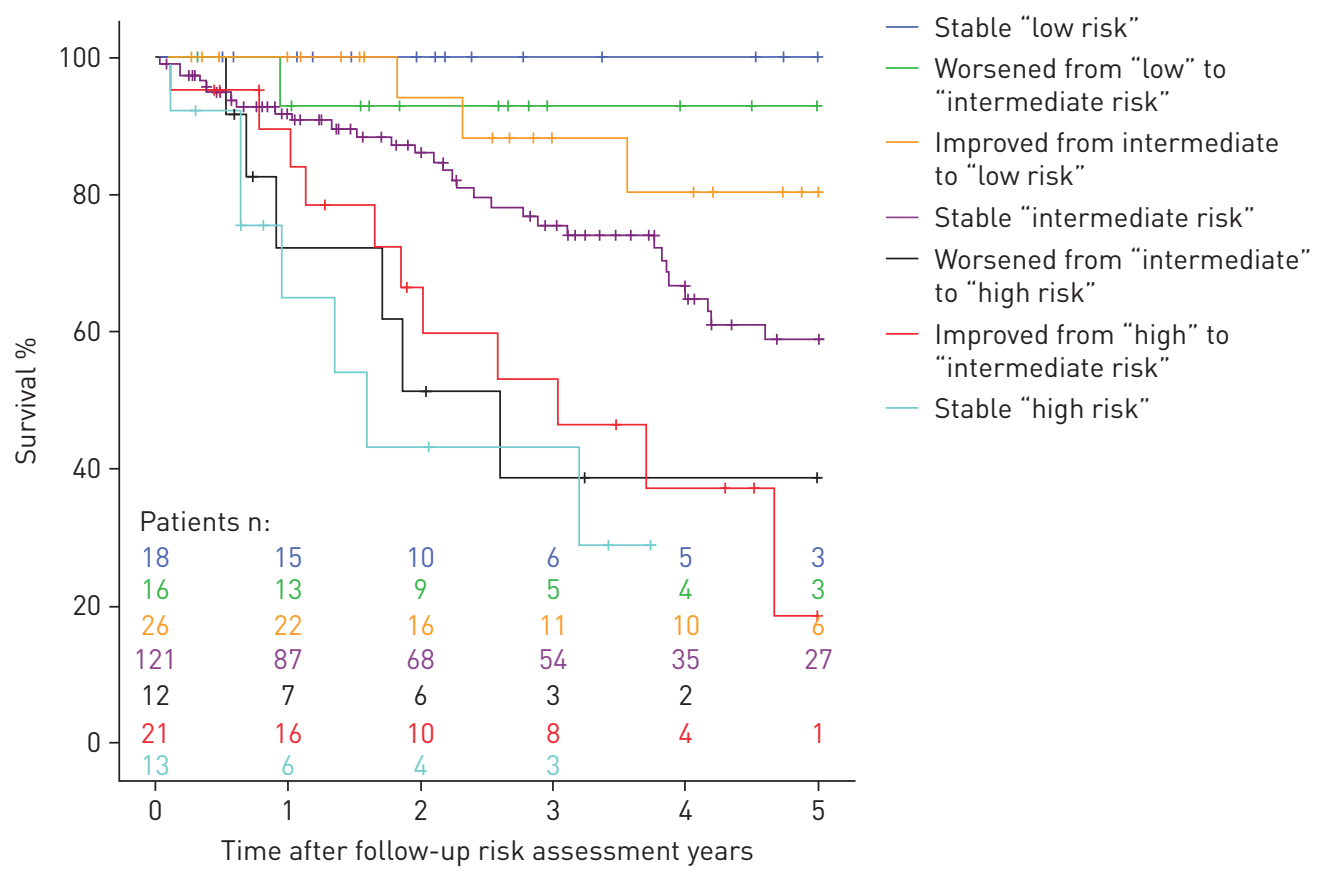

\begin{tabular}{|l|cccccc|}
\hline \multirow{2}{*}{ Survival \% } & \multicolumn{5}{c|}{ Years after enrolment } \\
\cline { 2 - 7 } & 0 & 1 & 2 & 3 & 4 & 5 \\
\hline Stable "low risk" & 100 & 100 & 100 & 100 & 100 & 100 \\
Worsened from "low" to "intermediate risk" & 100 & 92.9 & 92.9 & 92.9 & 92.9 & 92.9 \\
Improved from intermediate to "low risk" & 100 & 100 & 94.1 & 88.2 & 80.2 & 80.2 \\
Stable "intermediate risk" & 100 & 91.8 & 86.0 & 75.4 & 64.9 & 58.9 \\
Worsened from "intermediate" to "high risk" & 100 & 72.2 & 51.6 & 38.7 & 38.7 & \\
Improved from "high" to "intermediate risk" & 100 & 89.6 & 66.4 & 53.1 & 37.2 & 18.6 \\
Stable "high risk" & 100 & 64.7 & 43.2 & 43.2 & & \\
\hline
\end{tabular}

FIGURE 5 Five-year survival according to change in risk category from baseline to follow-up lat least three variables available).

similar functional class, exercise capacity and haemodynamics at diagnosis, of whom only $61 \%$ were treated with PAH medical therapy. Independent determinants of survival were WHO FC and RAP at diagnosis, together with the presence of comorbidities such as cancer, coronary disease, left heart failure and chronic obstructive pulmonary disease. There is further evidence supporting the prognostic relevance of most variables included in COMPERA ESC/ERS abbreviated risk score assessment. WHO FC [8, 9], 6MWD [10-12], RAP [9] and CI [10] were all shown to be independent prognostic factors, while $\mathrm{SvO}_{2}$ above the median was associated with better survival without being an independent survival predictor [10]. In agreement with current observations, 3-year survival ranged between $70 \%$ and $80 \%$ in the aforementioned nonsurgical cohorts. To the best of our knowledge, there are no registry data on the value of BNP/NT-proBNP as a prognostic indicator in CTEPH. Recently, an analysis of the 237 patients enrolled in CHEST-2 study (open-label follow-up of riociguat registration study [13]) showed that both 6MWD and NT-proBNP concentration at baseline and change from baseline to follow-up (but not absolute value at follow-up) were significantly and independently associated with survival. The association between WHO FC and survival was not significant in that study.

In the present series, $14 \%$ of the patients were in the low-risk group at baseline, which is similar to the previously PAH data published by the COMPERA investigators [4]. However, this proportion increased only minimally to $20 \%$ at follow-up (as opposed to PAH, where the proportion increased from $12 \%$ to $24 \%$ ). This may reflect the older age (69 versus 64 years) and more profound deconditioning of the CTEPH population, as well as the more restrictive use of combination therapy in CTEPH (only $7 \%$ versus $19 \%$ in $\mathrm{PAH}$ at baseline and $26 \%$ versus $41 \%$ at follow-up). Lack or limited efficacy of treatments with off-label PAH drugs (in 63\% of the patients) should also be considered. As in PAH $[2,4]$, the highest proportion of the patients was in the intermediate-risk group, both at baseline and at follow-up, which questions the need for a more refined approach to risk stratification. According to Cox regression, 6MWT, WHO FC and BNP/NT-proBNP were the 
a)

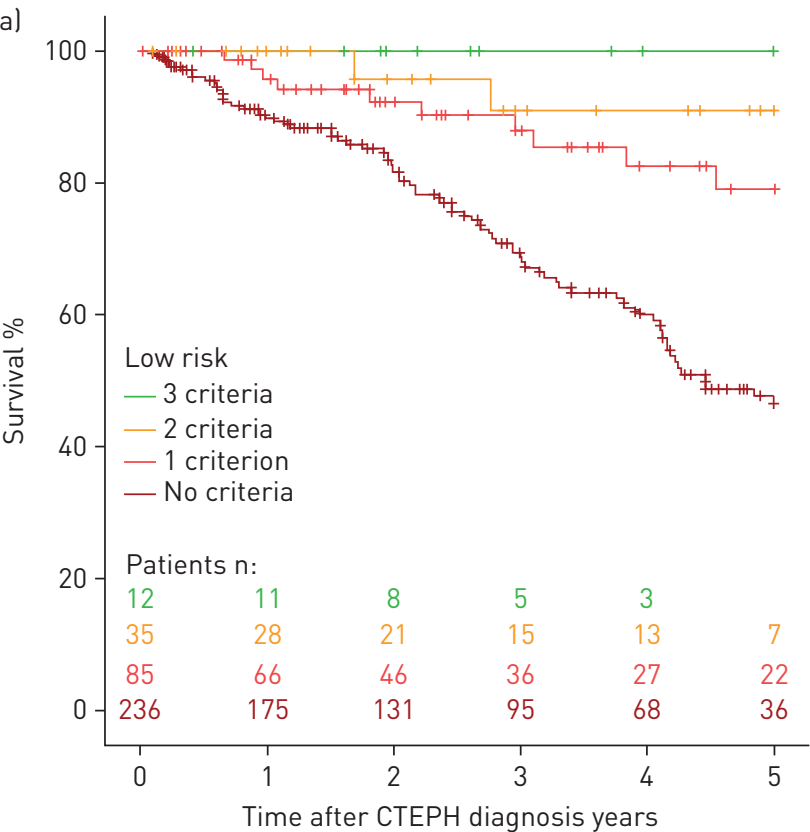

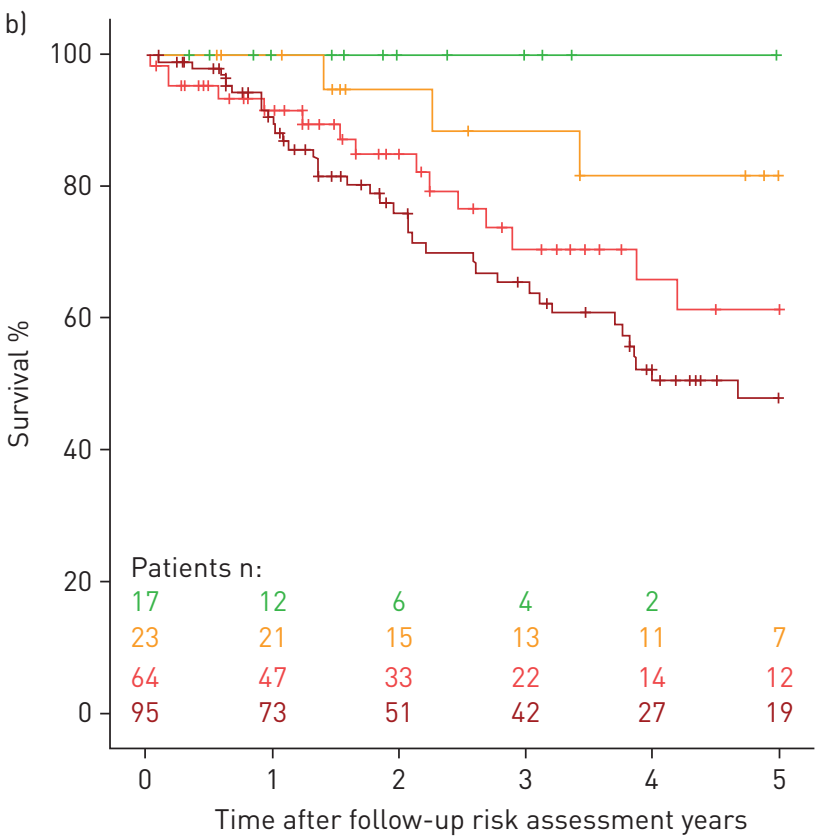

\begin{tabular}{|l|cccccc|}
\hline \multirow{2}{*}{ Survival \% } & \multicolumn{6}{|c|}{ Years after enrolment } \\
\cline { 2 - 7 } & 0 & 1 & 2 & 3 & 4 & 5 \\
\hline 3 criteria & 100 & 100 & 100 & 100 & 100 & \\
2 criteria & 100 & 100 & 94.7 & 88.4 & 81.6 & 81.6 \\
1 criterion & 100 & 91.6 & 84.8 & 70.5 & 65.8 & 61.1 \\
No criteria & 100 & 90.6 & 75.9 & 65.3 & 50.2 & 47.7 \\
\hline
\end{tabular}

FIGURE 6 Five-year survival according to the number of low-risk criteria: a) from baseline and b) from follow-up.

most discriminative variables, while only BNP/NT-proBNP and WHO FC were independent predictors of survival (figure 3 ).

The present study demonstrated that an abbreviated version of the ESC/ERS risk score assessment developed for PAH using at least three out of six selected variables provided accurate distinction between the risk groups in medically treated patients with CTEPH. Survival curves of the low- and intermediate-risk groups at baseline overlap during first 2 years, while the survival curves of the low- and intermediate-risk groups at follow-up separate immediately, underscoring the notion that risk assessment at follow-up, i.e. when patients receive medical therapies, allows for a more accurate prediction of survival than the baseline assessment. It is also possible that cut-off values and stratification strata suggested for $\mathrm{PAH}$, to segregate low- and intermediate-risk groups, do not perform as well in the CTEPH population. This is further illustrated by the sensitivity analysis involving only surgically inoperable patients, which showed better than expected 1-year survival in the low- and intermediate-risk groups (100\% and $98.8 \%$, respectively). While the Swedish approach performed reasonably well in discriminating the risk groups, the French noninvasive "risk score-3" at follow-up identified patients with an excellent long-term survival, similar to what has been reported in $\mathrm{PAH}[3,14]$. Unfortunately, we were not able to perform the French "risk score-4" analysis, including WHO FC, 6MWD, RAP and CI, because too few right heart catheterisations were performed at follow-up (RAP and CI available in only 33 patients).

In the present analysis, variables closely linked to mortality risk were 6MWD, WHO FC, BNP/ NT-proBNP and $\mathrm{SvO}_{2}$, whereas RAP and CI performed less well. Changes in the risk category, regardless of the direction, were predictors of long-term survival and may therefore be considered end-points in future clinical trials. In the present series, $34 \%$ of the patients with newly diagnosed CTEPH had experienced a change in risk category from baseline to follow-up, determined mainly by changes in WHO FC, 6MWD and BNP/NT-proBNP. Using this approach in CTEPH, we could also move from the short-term 6MWD/pulmonary vascular resistance trial design [15-17] to longer-term studies determining the net benefit, i.e. the ratio of patients who improve or worsen their risk category.

One of the most important limitations of our study was the fact that not all variables included in the risk stratification strategy proposed by the ESC/ERS pulmonary hypertension guidelines were available. 
Information on clinical signs of right heart failure, progression of symptoms, syncope, cardiopulmonary exercise tests and echocardiography were missing. Further limitations include missing values, especially haemodynamics at follow-up. When comparing risk assessment at baseline and at follow-up, we may argue that very severe patients might have died and very mild patients might have dropped out during the follow-up; however, there does not seem to be a significant selection bias, as shown by the overlap of patient characteristics between baseline and follow-up cohorts (supplementary table S3). No statistical measure was applied to account for the immortal time bias during the follow-up time window of 3-24 months (median 7 months), which would potentially have even enhanced the differences between the risk groups. Additionally, this study does not take into account the potential effects of BPA in inoperable CTEPH patients, since the technique has only recently been implemented in a limited number of European centres [18]. Still, in 2016, only $25 \%$ of the newly diagnosed inoperable CTEPH patients in Germany underwent BPA [19]. Even if we anticipate a further generalisation of the procedure, risk assessment is a dynamic concept and if BPA improves risk it will also improve outcomes with a better risk stratification at follow-up than at diagnosis, similar to what is observed in medically treated patients.

In conclusion, the current study shows that an abbreviated version of the ERS/ESC risk stratification may be applicable to medically treated CTEPH patients, with 1-year mortality rates conforming to the prediction ( $<5 \%$ for low risk, $5-10 \%$ for intermediate risk and $>10 \%$ for high risk). However, with the current therapeutic strategy largely based on monotherapy with off-label use of drugs not approved for the treatment of CTEPH, low risk is achieved in only one in five patients.

Acknowledgements: The authors are indebted to the COMPERA investigators.

Conflict of interest: M. Delcroix reports research grants, investigator, speaker and consultant fees from Actelion, investigator, speaker and consultant fees from Bayer and GSK, speaker and consultant fees from MSD, investigator fees from Reata and Eli Lilly, and investigator and consultant fees from Bellerophon, outside the submitted work. G. Staehler has received speaker's honoraria from Bayer and Actelion, during the conduct of the study. $\mathrm{H}$. Gall reports grants and personal fees from Actelion, AstraZeneca, Bayer, BMS, GSK, Janssen-Cilag, Lilly, MSD, Novartis, OMT, Pfizer and United Therapeutics, during the conduct of the study. E. Grünig reports grants and personal fees from Actelion, Bayer/ MSD, grants from GSK, grants from United Therapeutics, grants from Novartis, grants from Actelion, Bayer and MSD, personal fees from SCOPE, OrPha Swiss GmbH and Zurich Heart House, outside the submitted work. M. Held reports research grants and other from Actelion, honoraria for lectures from Actelion, Bayer Healthcare, Berlin Chemie, Boehringer Ingelheim, GSK, MSD, Novartis and Pfizer, participation in clinical trials for Actelion, Bayer, GSK, Pfizer and United Therapeutics, and honoraria for advisory board work from Actelion, Bayer, Boehringer, GSK and MSD, outside the submitted work. M. Halank reports personal fees and nonfinancial support from Actelion, Bayer, GSK, MSD, Novartis and OMT, outside the submitted work. H. Klose reports grants and personal fees from Bayer and Actelion, and personal fees from GSK, United Therapeutics and MSD, outside the submitted work. A. Vonk-Noordegraaf reports grants from NWO-VICI, speaker fees from Actelion and consultation fees from Arena, outside the submitted work. S. Rosenkranz reports grants and personal fees for speaking/consulting from Actelion, Bayer, Pfizer, Novartis and United Therapeutics, personal fees for speaking/consulting from GSK, Gilead and MSD, during the conduct of the study. J. Pepke-Zaba reports grants (compensation for data entry) from the COMPERA registry during the conduct of the study; grants, personal fees and nonfinancial support from Actelion, Bayer and Merck, outside the submitted work. C.F. Opitz reports other from Actelion, Bayer and MSD, outside the submitted work. J.S.R. Gibbs reports grants and personal fees from Actelion, Bayer, GSK and MSD, personal fees from Arena, Bellerophon and Pfizer, and grants from United Therapeutics, during the conduct of the study. T.J. Lange reports grants (compensation for data entry) from the COMPERA registry, during the conduct of the study; grants, personal fees and nonfinancial support from Actelion, Bayer, GSK and Pfizer, personal fees and nonfinancial support from AOP Orphan Pharmaceuticals and MSD, and grants and personal fees from United Therapeutics, outside the submitted work. I. Tsangaris reports grants, personal fees and nonfinancial support from Actelion, GSK and Bayer, and personal fees and nonfinancial support from MSD and United Therapeutics, outside the submitted work. D. Huscher reports personal fees from Actelion, outside the submitted work. D. Pittrow reports personal fees from Actelion, Bayer, Aspen, Boehringer Ingelheim, AstraZeneca and MSD, outside the submitted work. K.M. Olsson reports personal fees for lectures from Actelion, Bayer, GSK, Pfizer and United Therapeutics, outside the submitted work. M.M. Hoeper reports personal fees for lectures and consultations from Actelion, Bayer, Gilead, GSK, MSD and Pfizer, outside the submitted work.

Support statement: COMPERA is funded by unrestricted grants from Actelion Pharmaceuticals, Bayer, GSK and OMT. These companies were not involved in data analysis or the writing of this manuscript. Funding information for this article has been deposited with the Crossref Funder Registry.

\section{References}

1 Galiè N, Humbert M, Vachiéry J-L, et al. 2015 ESC/ERS Guidelines for the diagnosis and treatment of pulmonary hypertension. The Joint Task Force for the Diagnosis and Treatment of Pulmonary Hypertension of the European Society of Cardiology (ESC) and the European Respiratory Society (ERS). Eur Respir J 2015; 46: 903-975.

2 Kylhammar D, Kjellstrom B, Hjalmarsson C, et al. A comprehensive risk stratification at early follow-up determines prognosis in pulmonary arterial hypertension. Eur Heart J 2017; 40: 596.

3 Boucly A, Weatherald J, Savale L, et al. Risk assessment, prognosis and guideline implementation in pulmonary arterial hypertension. Eur Respir J 2017; 50: 1700889. 

European pulmonary hypertension guidelines risk stratification model. Eur Respir J 2017; 50: 1700740.

5 Hoeper MM, Huscher D, Ghofrani HA, et al. Elderly patients diagnosed with idiopathic pulmonary arterial hypertension: results from the COMPERA registry. Int J Cardiol 2013; 168: 871-880.

6 Opitz CF, Hoeper MM, Gibbs JSR, et al. Pre-capillary, combined, and post-capillary pulmonary hypertension: a pathophysiological continuum. J Am Coll Cardiol 2016; 68: 368-378.

7 Delcroix M, Lang I, Pepke-Zaba J, et al. Long-term outcome of patients with chronic thromboembolic pulmonary hypertension: results from an international prospective registry. Circulation 2016; 133: 859-871.

8 Skoro-Sajer N, Bonderman D, Wiesbauer F, et al. Treprostinil for severe inoperable chronic thromboembolic pulmonary hypertension. J Thromb Haemost 2007; 5: 483-489.

9 Wieteska M, Biederman A, Kurzyna M, et al. Outcome of medically versus surgically treated patients with chronic thromboembolic pulmonary hypertension. Clin Appl Thromb Hemost 2016; 22: 92-99.

10 Condliffe R, Kiely DG, Gibbs JSR, et al. Prognostic and aetiological factors in chronic thromboembolic pulmonary hypertension. Eur Respir J 2009; 33: 332-338.

11 Saouti N, de Man F, Westerhof N, et al. Predictors of mortality in inoperable chronic thromboembolic pulmonary hypertension. Respir Med 2009; 103: 1013-1019.

12 Scholzel BE, Post MC, Thijs Plokker HW, et al. Clinical worsening during long-term follow-up in inoperable chronic thromboembolic pulmonary hypertension. Lung 2012; 190: 161-167.

13 Simonneau G, D'Armini AM, Ghofrani HA, et al. Predictors of long-term outcomes in patients treated with riociguat for chronic thromboembolic pulmonary hypertension: data from the CHEST-2 open-label, randomised, long-term extension trial. Lancet Respir Med 2016; 4: 372-380.

14 Hoeper MM, Pittrow D, Opitz C, et al. Risk assessment in pulmonary arterial hypertension. Eur Respir J 2018; 12: 1702606.

15 Jais X, D’Armini AM, Jansa P, et al. Bosentan for treatment of inoperable chronic thromboembolic pulmonary hypertension: BENEFiT (Bosentan Effects in iNopErable Forms of chronIc Thromboembolic pulmonary hypertension), a randomized, placebo-controlled trial. J Am Coll Cardiol 2008; 52: 2127-2134.

16 Ghofrani HA, D'Armini AM, Grimminger F, et al. Riociguat for the treatment of chronic thromboembolic pulmonary hypertension. N Engl J Med 2013; 369: 319-329.

17 Ghofrani HA, Simonneau G, D'Armini AM, et al. Macitentan for the treatment of inoperable chronic thromboembolic pulmonary hypertension (MERIT-1): results from the multicentre, phase 2, randomised, double-blind, placebo-controlled study. Lancet Respir Med 2017; 5: 785-794.

18 Olsson KM, Wiedenroth CB, Kamp J-C, et al. Balloon pulmonary angioplasty for inoperable patients with chronic thromboembolic pulmonary hypertension: the initial German experience. Eur Respir J 2017; 49: 1602409.

19 Kramm T, Wilkens H, Fuge J, et al. Incidence and characteristics of chronic thromboembolic pulmonary hypertension in Germany. Clin Res Cardiol 2018; 107: 548-553. 